\title{
A tactile metacontrast effect
}

\author{
JANET M. WEISENBERGER and JAMES C. CRAIG \\ Indiana University, Bloomington, Indiana
}

\begin{abstract}
Vibrotactile patterns, generated on the $6 \times 24$ array of the Optacon, were presented to subjects' left index fingertips. The subjects identified these patterns in the absence of any masking stimuli and in the presence of spatially adjacent masking stimuli. The amount of interference in recognizing the patterns was measured as a function of the interval between target and masker onsets. Masking functions similar to those reported in visual metacontrast studies were found; that is, more masking occurred when masker onset followed target onset by 25 to 50 msec than when onsets were simultaneous. Subjects showed more metacontrast when masker energy was reduced, a finding paralleled in the visual literature. The relevance of some models of visual metacontrast to the tactile findings is discussed.
\end{abstract}

\begin{abstract}
"Stages of processing" models as descriptions of the response to sensory input have been commonly accepted for the visual and auditory modalities. Relatively few studies have investigated stages of processing models with cutaneous stimuli, and even fewer have examined the initial stages of processing and storage in the tactile modality. This lack is in part due to the fact that much of the research into the initial response of the visual and auditory systems to stimuli has been concerned with relatively fast processes. Comparisons with tactile studies have proved difficult, because of the relatively long times usually required for controlled cutaneous pattern generation.
\end{abstract}

The recent development of sophisticated sensory prostheses makes comparisons of tactile and visual stimulus processing more feasible than in the past. The Optacon (Bliss, Katcher, Rogers, \& Shepard, 1970 ) is particularly useful in this regard, because it transmits printed text rapidly to the observer's fingertip via a tactile array of vibrating pins. A camera on the Optacon is passed over a letter, and the array produces a pattern of vibration in the shape of the letter, allowing the observer to "read" with his fingertip. The Optacon has also been interfaced with a computer, an arrangement that can be used to present stimuli that are quantitatively analogous to the stimuli used in visual experiments and permits a high degree of comparability of visual and tactile processing (Craig, 1980).

\footnotetext{
This research was supported by Grant NS-09783 from the National Institutes of Health. A portion of these results was presented at the 1980 meeting of the Midwestern Psychological Association. The authors wish to thank Roger Rhodes for his assistance with programming and data collection. Address correspondence to: James C. Craig, Department of Psychology, Indiana University, Bloomington, Indiana 47405. J. M. Weisenberger's present address is: Central Institute for the Deaf, $818 \mathrm{~S}$. Euclid, St. Louis, Missouri 63110.
}

Previous studies have examined several modes of generating tactile patterns and the effects that masking stimuli have on letter recognition. Two findings from these studies are particularly relevant to the present study. First, the presentation of a masking stimulus following the target, a backward masker, produces consistently more interference than presentation of the masker before the target, a forward masker (Craig, 1976, 1978, 1980). This greater amount of backward than of forward masking parallels the findings from visual pattern recognition studies. Second, the so-called "static" mode of generating tactile patterns (turning on all elements in the pattern simultaneously) has proven to be a very effective way of presenting patterns at brief durations. Because good letter recognition with the static mode is possible for pattern durations as brief as $4 \mathrm{msec}$, and because this mode is very similar to the mode employed with visual stimuli, it is possible to compare the two modalities quantitatively.

In previous studies of tactile masking using the Optacon display, the masking stimulus either preceded or followed the target, but was not presented at the same time as the target. Most of the forward/ backward masking functions generated showed maximum interference with letter recognition when the offset of the target was coincident with the onset of the masker, that is, a backward masker with a 0 -msec interstimulus interval. This result suggests that even more interference might be obtained if a masking stimulus were presented simultaneously with the target.

However, Craig (Not: 1) has recently found some evidence suggesting that, for some conditions, minimum performance occurs with a backward masker that follows the target by some time interval. With a brief (4-msec) static presentation of letters followed by a 4-msec masker, the maximum amount of masking occurred when the interval between letter and 
masker onsets was $75 \mathrm{msec}$. At longer stimulus onset asynchronies (SOAs), performance improved.

The possibility that a U-shaped backward masking function could be obtained on the skin brings to mind similar functions that have been produced using visual stimuli. The paradigm under which such functions are generated involves a target masked by spatially adjacent contours presented at various temporal separations from the target, the result being the phenomenon known as metacontrast. The exact temporal asynchrony at which maximum masking occurs is determined by the duration, configuration, and placement of the visual stimuli.

Visual researchers have extensively investigated the phenomenon of metacontrast, using a variety of stimulus configurations. Alpern's (1953) early work found U-shaped functions with simple rectangles of light masked by adjacent rectangular contours. Later studies reported similarly shaped functions when more complex tasks were used; Spencer and Shuntich (1970), for example, required subjects to identify letters of the alphabet, as did Turvey (1973). Turvey showed further that metacontrast functions were obtained when target energy was greater than masker energy; if the masker had more energy than the target, performance was essentially a monotonic function of the interval between target and masker onsets. To explain this difference in performance, Turvey postulated the existence of both peripheral and central processes, with the metacontrast effect resulting from interactions at the central stage. Uttal (1971) argued for a central locus" for visual metacontrast effects, finding a larger effect when the shapes of the target and masker were similar. According to his argument, some preliminary form processing must be occurring prior to the masking effect for such selective interference to take place.

A number of theories have been proposed to explain visual metacontrast phenomena (Alpern, 1953; Crawford, 1947; Weisstein, 1972). One feature common to most of these explanations is their postulation of an inhibitory neural trace generated by the masker that develops more quickly than the excitation generated by the target. Metacontrast effects are explained in the context of a larger theory of visual masking developed by Breitmeyer and Ganz (1976). They argue, based on the relatively retinotopic nature of the stimulus representation in metacontrast, that the effect occurs at a locus peripheral to the loss of retinotopic information. They attempt to explain visual masking effects in terms of inhibition within and between sustained and transient channels at subcortical and primary cortical levels.

At the present time, all of these explanations of masking phenomena have been described specifically in reference to the visual system, although some of the mechanisms proposed could be generalized to include other systems. Given the suggestion that the skin's information processing characteristics are sim- ilar in some respects to those of the visual system, an expansion of theoretical explanations of visual masking to encompass the tactile system might provide a useful approach for comparing the functioning of both modalities.

The present study examines the performance of the tactile system when presented with vibrotactile patterns and maskers in a stimulus configuration similar to that used in visual metacontrast studies. The target patterns were six geometric shapes presented on the middle of the Optacon array. The masking stimulus consisted of a pair of bars located above and below the target on the array. The masker was presented before, simultaneously with, or after the target. Even if explanations of visual processing under such conditions should prove inadequate for the tactile system, the study of these conditions should lead to a better understanding of the early stages of tactile stimulus processing.

\section{EXPERIMENT 1}

\section{Method}

Subjects. Four undergraduate students (three women and one man) were paid an hourly wage for their participation in the study. They had previously passed a screening test designed to identify persons who can quickly reach a high level of asymptotic performance in tasks involving the Optacon (Craig, 1980). In addition, these subjects had served in previous research using the Optacon for letter identification.

Apparatus. Stimuli were presented to the top $2.7 \mathrm{~cm}$ of the subject's left index fingertip using the Optacon, a reading aid for the blind manufactured by Telesensory Systems, Inc. The Optacon contains a display, $2.7 \times 1.1 \mathrm{~cm}$, of 144 pins upon which the fingertip is placed. The pins are arranged in a 6 column by 24 row array. When activated, each pin vibrates at a frequency of about $230 \mathrm{~Hz}$. In the present study, patterns were generated on the tactile array by a PDP-11/34 computer interfaced to the Optacon. The computer permitted individual control of each pin of the array. In addition to presenting the tactile patterns, the computer recorded responses and presented feedback.

Target stimuli were six geometric shapes (Figure 1) presented
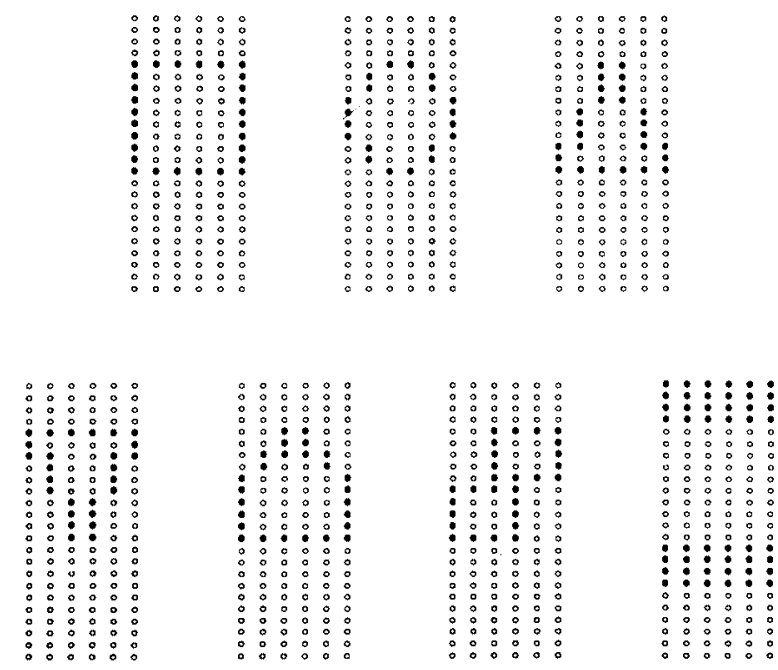

Figure 1. Patterns used as target and masking stimuli in Experiment 1. 
on rows 5-14 and all six columns of the Optacon array. The masking stimulus consisted of two horizontal bars that were produced by activating four rows and all six columns for each bar. The bars were located immediately above (rows 1-4) and below (rows 15-18) the target, as shown in Figure 1. Both target and masker were presented for a duration of $26 \mathrm{msec}{ }^{1}$ The masker was activated at various times before, concurrently with, or after the target presentation. The intensity of both the target and the masker was set at $33 \mathrm{~V}$ to the Optacon display, which produced a comfortable level of vibration.

Procedure. On each trial, a cue stimulus, consisting of a horizontal line occupying row 12 and all six columns of the array for $12 \mathrm{msec}$, preceded the target by $1 \mathrm{sec}$. The subjects identified the target pattern by pressing a key on a keyboard; they were then informed on a visual monitor which pattern had been presented.

Pattern recognition was tested at nine intervals between the onsets of target and masker (SOA) and in the absence of a masker. The SOAs tested were $-129,-56,-30,0,30,56,129,229$, and $429 \mathrm{msec}$, where negative values refer to conditions in which the masker preceded the target (forward masking) and positive values indicate simultaneous or backward masking.

Subjects received 300 trials in a daily testing session in which all SOAs were tested. The sessions consisted of a series of 30-trial blocks arranged such that all trials within a block tested the same SOA. A total of 360 trials from each subject was obtained for each SOA, making a total of 1,440 trials per SOA. To reduce auditory cues and distractions during testing, the subjects wore earphones through which white noise was fed.

\section{Results and Discussion}

The results of Experiment 1 are shown in Figure 2. Each point represents 360 trials from each of four subjects, 1,440 trials per point. As noted before, previous measures of forward and backward tactile recognition masking had used spatially overlapping stimuli and had not presented the masking stimuli simultaneously with the target. Whereas these previous measures had found considerably more backward than forward masking, it was not possible to state at what SOA maximum interference would occur. The present results show that maximum interference in pattern recognition under these conditions

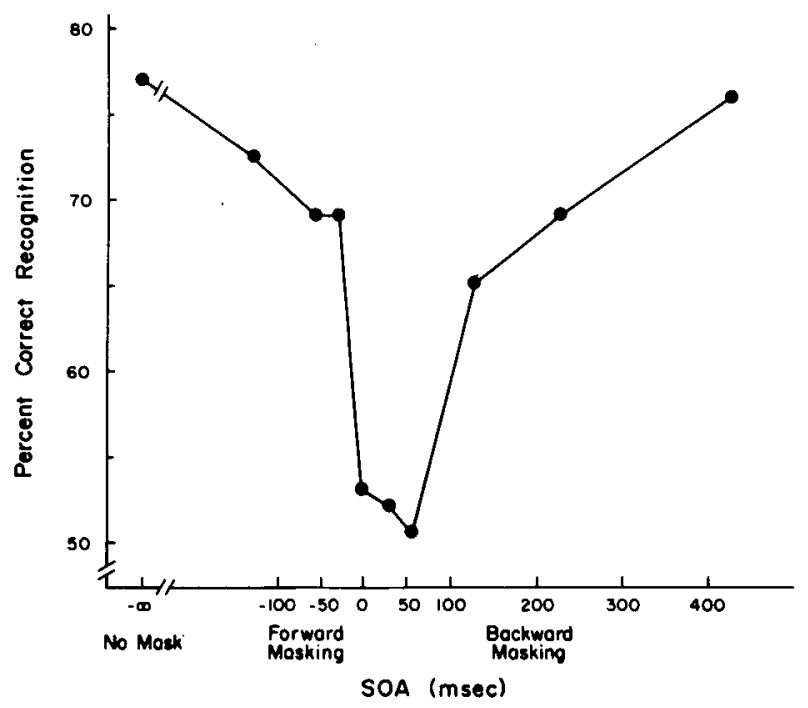

Figure 2. Percent correct pattern identification as a function of SOA. is not, as might be predicted from previous measures, acheived when the target and masker are presented simultaneously. In fact, the results show minimum performance at an SOA of $56 \mathrm{msec}$, a result very similar to that found with visual stimuli in metacontrast studies. The difference between the minimum point at $56 \mathrm{msec}$ and at $0 \mathrm{msec}$ is small and fails to reach statistical significance. Experiment 2 considers possible reasons why this difference is as small as it is, and demonstrates how the difference can be increased.

One possible explanation for the small size of the metacontrast effect in Experiment 1 involves the intensity relations of target and masker. In the visual literature, Turvey (1973) found metacontrast effects when the target intensity exceeded that of the masker (target-to-masker ratio of 2:1). As masker intensity increased relative to the target, the effect weakened, and when the target-to-masker intensity ratio was $1: 2$, maximum masking occurred when target and masker were presented simultaneously. In Experiment 1 of the present study, it could be that the masker was too intense to show a strong metacontrast effect.

It has been suggested (Turvey, 1973) that the effect of masker intensity on metacontrast masking can be explained in terms of a model of forward and backward masking that posits the two processes of integration and interruption. Masking resulting from integration is considered to be dependent on masker energy, whereas interruption masking is dependent on temporal factors and is relatively independent of masker energy. According to this explanation, high-intensity maskers produce a large amount of peripheral masking, causing the masker to be integrated with the target and resulting in more symmetric forward and backward masking functions. This greater amount of integration masking could obscure the interruption of target processing that occurs with backward maskers at longer temporal separations. When masker intensity is not as great, both integration and interruption effects appear, and these processes are reflected in the U-shaped metacontrast function.

A two-process model of metacontrast masking would appear to be necessary to account for the asymmetry of forward and backward masking functions. Models positing a single integrative mechanism predict symmetric forward and backward masking effects. Simple interruptive theories, moreover, cannot account for forward masking effects at all. The observed asymmetry of these effects indicates that more than one process mediates masking of visual patterns. Perhaps the most compelling feature of a two-process integration and interruption model is its ability to predict asymmetric forward and backward masking functions.

However, more recent theoretical formulations (Eriksen, 1980; Felsten \& Wasserman, 1980) have 
argued that many of the effects attributed to the operation of interruption-like processes can be explained equally well by integration. These recent arguments note that to date no evidence has been found on the physiological level to support the existence of interruption masking, and suggest, instead, ways in which physiological integrative mechanisms can produce psychophysical effects that appear to be interruptive in nature. The asymmetry of forward and backward masking functions, together with the results from masking studies using dichoptic presentation, have led researchers to postulate different peripheral and central integrative masking effects. Physiological results such as those of Bridgeman (1975) have offered evidence for such differentiation on the cortical level. Bridgeman, using a metacontrast paradigm, recorded from cells in the cat cortex that showed a two-component response. The second peak of the response was reduced maximally at masker SOAs of $60 \mathrm{msec}$. This response was not found at lower levels in the system, such as the optic tract and lateral geniculate nucleus. Thus one current approach to explaining visual masking effects postulates physiological integrative effects that occur at several levels in the system. The observed backward masking function results from target-masker interactions whose properties differ as a function of SOA.

Turvey's (1973) finding that the shape of the masking function in metacontrast paradigms depends on the relative intensities of target and masker may thus reflect the operation of integrative effects on several levels of the visual system (Felsten \& Wasserman, 1980). Even a brief examination of the visual literature on metacontrast and visual masking in general indicates that there is no agreement on the specific mechanisms underlying these phenomena. For the purposes of the present study, this lack of agreement among visual researchers does not diminish the interest of empirical comparisons between visual and tactile pattern masking effects. Indeed, such comparisons may shed some light on the mechanisms underlying visual masking. Whatever the theoretical explanation for Turvey's finding, it was thought that the results of Experiment 1 of the present study might show a similar change with manipulations of masker energy. Experiment 2 therefore measured the effect of reduced masker energy on pattern recognition.

\section{EXPERIMENT 2}

Experiment 2 studied the effect on pattern recognition of two "reduced" maskers. First, the fourrow maskers of Experiment 1 were replaced by maskers occupying two rows above and below the target. These maskers were further reduced to one row above and below the target. Thus, the total number of pins activated on the array by the masking stimulus was reduced from 48 in Experiment 1 to 24 and then to 12 in Experiment 2. It might be expected that reduction of masker energy by reducing the number of rows would create a stronger metacontrast effect by virtue of the lesser effect of the masker on the target when presented simultaneously with it. In other words, performance with a reduced masker might be generally improved, but show less improvement with a backward masker presented at some time after the target. Thus, a larger difference is expected between an SOA of $0 \mathrm{msec}$ and the point of minimum performance.

The above-described manipulation might appear less straightforward, and possibly less preferable, than a simple manipulation of the intensity of the vibration of the pins making up the masker. However, the interface linking Optacon and computer used in the present study does not currently permit such direct and precise control of the intensity of patterns presented on the tactile array. For this reason, changes in the number of pins making up the masker were selected to study the effect of masker intensity on pattern recognition.

\section{Method}

Subjects. Four undergraduate students, two men and two women, were paid for their participation in the experiment. All had previously served as subjects in experiments using the Optacon. None of the four subjects had participated in Experiment 1.

Apparatus. The apparatus used was the same as that of Experiment 1. Target stimuli were the six shapes used in Experiment 1, again presented for $26 \mathrm{msec}$. Two maskers were used. The first (two-row) masker consisted of two bars located directly above (rows 3-4) and below (rows 15-16) the target, each bar extending across two rows of the array. The second (one-row) masker consisted of one-row bars located in rows 4 and 15 of the array.

Procedure. The general procedure was the same as that of Experiment 1, with the following exception: The cue stimulus for Experiment 2 consisted of the 50-msec activation of the two pins on the top row of the array corresponding to columns 1 and 6. This cue stimulus also served as an aid for maintaining finger placement on the array.

Pattern recognition was tested in the absence of a masker, and at SOAs of 0,29 , and $56 \mathrm{msec}$. As before, the positive SOA values indicate simultaneous or backward masking.

Subjects received 320 trials per daily testing session. The trials were arranged in 40-trial blocks, with all trials in a block having the same SOA. Testing was completed with the two-row masker before testing was begun with the one-row masker.

\section{Results and Discussion}

The results of the measurements for both maskers are shown in Figures 3 and 4. A total of 160 trials per subject was obtained for each SOA with each masker, for a total of 640 trials per SOA for each masker. Figure 3 shows performance of the individual subjects, and Figure 4 presents the average of the four subjects.

The data for the two-row maskers in Figure 4 reflect a larger change in average performance between 

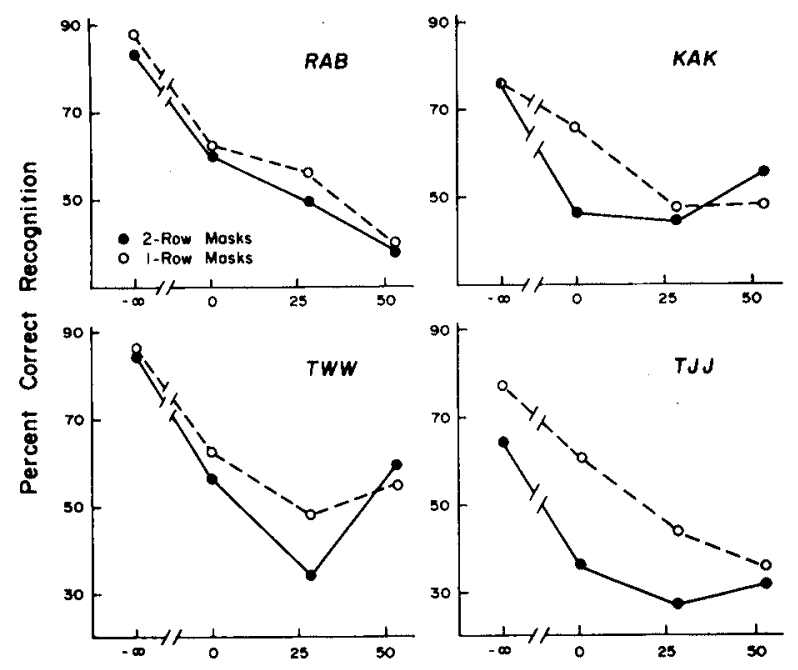

SOA (msec)

Figure 3. Percent correct pattern identification as a function of SOA. Maskers of two different intensities were used. Data from each subject are shown separately.

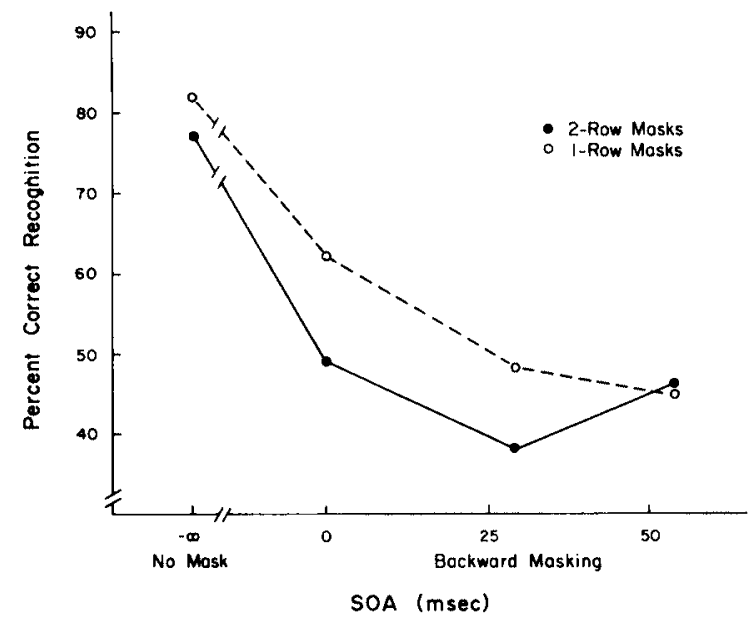

Figure 4. Results from Figure 3 showing mean performance from four subjects.

SOAs of 0 and $29 \mathrm{msec}$ than the change between the same SOAs in Figure $2(11 \%$ vs. $1 \%)$. For the onerow masker, the change between these two SOA values is even larger $(14 \%)$. The one-row maskers yielded a decrease in performance of $17 \%$ from an SOA of 0 to an SOA of $56 \mathrm{msec}$ as compared with the $3 \%$ difference produced by the four-row masker of Experiment 1 (Figure 2). Interestingly, the tworow masker data show a performance minimum for the times tested at an SOA of $29 \mathrm{msec}$. By comparison, the minimum for the one-row masker for the times tested, as well as the masker of Experiment 1 , occurs at 56-msec SOA. The reason for this difference could be due to the wide individual variations in performance, as shown in Figure 3. However, both curves in Figure 4 show clear metacontrast effects that are considerably larger than that reflected in Figure 2.

Thus, it appears that recovery from masking as the SOA is lengthened does not occur for the conditions using one- and two-row maskers. However, in comparing the amount of masking for any SOA generated by the one- and two-row maskers with the amount generated by the four-row masker in Experiment 1 , it is puzzling to note that subjects did not show less masking overall with the lower energy maskers, even at an SOA of 0 msec (Figures 2 and 4). There are several possible explanations for such a result. First, the two experiments were performed with different subjects, making the direct comparability of specific values of the functions infeasible.

A second possibility is that the perceived intensity of the reduced maskers might not be radically different from that of the four-row masker used in Experiment 1 . Preliminary results by Green (Note 2) with a magnitude estimation task suggest that the perceived intensity of $26-\mathrm{msec}$ stimuli presented on the Optacon increases as the number of columns (24 pins each) or rows (6 pins each) in the stimulus is increased. The function relating perceived intensity to number of pins is a power function with a slope of about .6. Green's data suggest that there would be a change in perceived intensity for the masking stimuli used in the present study, but that halving the number of pins is unlikely to halve the perceived intensity of the masker. It should be pointed out that the maskers used in the present study differed from the stimuli presented by Green, in that the top and bottom portions of the maskers were separated by 10 rows of the tactile array. This separation could have altered the perceived intensity of the masking stimuli. In addition, the perceived intensity of the masker when presented with a target stimulus might be very different from its perceived intensity when presented alone. This difference would be difficult to measure using conventional magnitude estimation, owing to the problem of having subjects estimate the intensity of parts of the array while attempting to ignore a stimulus presented in the middle of the array. Green's data further suggest that the slope of the magnitude estimation functions for stimuli ranging from one to five columns of the array increases as stimulus duration increases. Thus, somewhat different masking results might be expected for maskers of longer duration.

Another possible explanation for the lack of change in overall amount of masking with the reduced maskers is that the one- and two-row maskers were in some way perceived as more "similar" to the tar- 
gets, and thus interfered with target identification on that basis, obscuring decreases in masking from the four-row masker condition that would presumably occur as masker intensity was decreased. The result could also be interpreted as a characteristic of the tactile system that does not parallel the results for similar visual stimuli. As noted above, there is some disagreement on the precise mechanism mediating visual metacontrast effects. To a certain extent, the prediction one might make for the system's response to changes in intensity depends on the mechanism or model proposed to explain the system's operation. Pending further investigation, it may be most prudent for the present time to simply note the observed discrepancy between the visual findings and the results of the present study.

The standard errors of the points plotted in Figure 4 range from $2.5 \%$ to $3.5 \%$. The strength of the metacontrast effect for the two curves in Figure 4 was examined by comparing performance with a masker with an SOA of $0 \mathrm{msec}$ with minimum performance for each curve. The decrease in performance for the two-row maskers from an SOA of $4 \mathrm{msec}$ to an SOA of $29 \mathrm{msec}$ is statistically significant $[t(15)=2.76, p<.02]$. Likewise, the drop for the one-row masking curve from an SOA of $4 \mathrm{msec}$ to an SOA of $56 \mathrm{msec}$ is also significant $[\mathrm{t}(15)=$ $4.22, \mathrm{p}<.001$ ]. The improvement in performance occurring in the absence of a masker for the onerow masker curve over the two-row curve shown in Figure 4 can be attributed to a slight learning effect.

Figure 3 shows considerable variation among individual subjects in the shape of their masking functions, as stated above. This variation is consistent with findings in visual studies (Breitmeyer \& Ganz, 1976; Weisstein, 1972). In general, Figure 3 indicates that, for most subjects, the effect of reducing the masker from two rows to one row appears to be an overall improvement in performance, with little or no improvement at an SOA equal to $56 \mathrm{msec}$. This tendency for the point of maximum masking to shift toward longer SOAs as the masker is reduced from two rows to one row could be interpreted as a shift toward a more metacontrast-like function.

\section{GENERAL DISCUSSION}

The results of Experiments 1 and 2 indicate that the tactile system shows metacontrast effects paralleling those found in the visual system. These results support previous tactile research suggesting the existence of similarities between tactile and visual processing of complex stimuli. In the present study, the stimuli showed not only maximum masking with a nonsimultaneous masker, but also changes in the effect with changes in the ratio of target intensity to masker intensity.
The extent to which the two modalities process patterns in a similar manner has not been examined in detail. Clearly, the skin lacks the ability for spatial resolution found in the visual system. However, several studies (e.g., Kolers \& Rosner, 1960) indicate that effects such as visual metacontrast are found primarily in the periphery of the retina and occur only minimally in the fovea. The lower acuity of the peripheral retina might make it more comparable to the more highly sensitive tactile areas such as the fingertip, and leads to the speculation that even greater metacontrast effects might be found if less sensitive areas of the body were tested. Alternatively, one might present smaller patterns to the fingertip, taxing that area's spatial resolution ability and enhancing the metacontrast effect in this way.

Because the present study represents some of the first steps in drawing particular parallels between the tactile and visual modalities, it is not surprising that there remains a considerable amount of experimentation to be done to define the areas in which such parallels can be drawn. It is also not surprising that tactile results can be obtained that either do not have a parallel in the visual literature or are not predicted by current views of metacontrast. Some measurements were made in our laboratory with tactile patterns presented for durations of $52 \mathrm{msec}$ and $9 \mathrm{msec}$. The $52-\mathrm{msec}$ results showed a minimum in the masking function at an SOA of $56 \mathrm{msec}$, that is, a metacontrast effect. For the 9-msec patterns, the minimum was at $0 \mathrm{msec}$, a simultaneous presentation of target and masker. Metacontrast effects can be obtained with brief visual stimuli (Spencer \& Shuntich, 1970). If these preliminary results for 9-msec tactile patterns were to hold up under further testing, it might mean that there is a basic difference in the processing time for cutaneous as opposed to visual patterns.

In addition, results with visual stimuli have shown that targets that are very different in form from the masker show the most masking when target and masker onset are simultaneous, whereas similar targets and maskers show greatest interference with backward maskers, a U-shaped masking function (Uttal, 1970, 1971). An analysis of the results from Experiments 1 and 2 of the present study showed that different patterns showed minima at different SOAs. Some patterns showed minimum performance at an SOA of $0 \mathrm{msec}$, others did so at $29 \mathrm{msec}$, and still others at $56 \mathrm{msec}$. However, these minima are not predictable on the basis of the visual similarity of target and masker. While these findings may indicate that the tactile system does not parallel the visual system with respect to target and masker similarity, it is just as likely that tactile similarity is not identical to visual similarity. This issue is currently being investigated in our laboratory. Direct measurements of tactile similarity will be required 
to resolve this issue, as, indeed, additional research will be necessary to determine the extent to which further parallels may be drawn between tactile and visual processing.

\section{REFERENCE NOTES}

1. Craig, J. C. Pattern and energy masks in vibrotactile letter recognition. Paper presented at the meeting of the Psychonomic Society, St. Louis, Missouri, November 1980.

2. Green, B. G. Perceived magnitude as a function of duration and size for stimuli presented on the Optacon. Manuscript in preparation, 1981 .

\section{REFERENCES}

AlPERn, M. Metacontrast. Journal of the Optical Society of America, 1953, 43, 648-657.

Bliss, J. C., Katcher, M. H., Rogers, C. H., \& Shepard, R. P. Optical-to-tactile image conversion for the blind. IEEE Transactions on Man-Machine Systems, 1970, MMS-11, 58-64.

Breitmeyer, B. G., \& GANZ, L. Implications of sustained and transient channels for theories of visual pattern masking, saccadic suppression, and information processing. Psychological Review, 1976, 83, 1-36.

Bridgeman, B. Correlates of metacontrast in single cells of the cat visual system. Vision Research, 1975, 15, 91-99.

Craig, J. C. Vibrotactile letter recognition: The effects of a masking stimulus. Perception \& Psychophysics, 1976, 20, 317-326.

Craig, J. C. Vibrotactile pattern recognition and masking. In G. Gordon (Ed.), Active touch-the mechanism of recognition of objects by manipulation-a multidisciplinary approach. Oxford: Pergamon Press, 1978.

Craig, J. C. Modes of vibrotactile pattern generation. Journal of
Experimental Psychology: Human Perception and Performance, $1980,6,151-166$.

Crawford, B. H. Visual adaptation in relation to brief conditioning stimuli. Proceedings of the Royal Society, London, 1947, 134B, 283-302.

ERIKSEN, C. W. The use of a visual mask may seriously confound your experiment. Perception \& Psychophysics, 1980, 28, 89-92.

Felsten, G., \& Wasserman, G. S. Visual masking: Mechanisms and theories. Psychological Bulletin, 1980, 88, 329-353.

Kolers, P. A., \& Rosner, B. S. On visual masking (metacontrast): Dichoptic observations. American Journal of Psychology, 1960, 73, 2-21.

Spencer, T. J., \& Shuntich, R. Evidence for an interruption theory of backward masking. Journal of Experimental Psychology, 1970, 85, 198-203.

TuRveY, M. T. On peripheral and central processes in vision: Inferences from an information processing analysis of masking with patterned stimuli. Psychological Review, 1973, 80, 1-52.

UTTAL, W. R. On the physiological basis of masking with dotted visual noise. Perception \& Psychophysics, 1970, 7, 321-327.

UTTAL, W. R. The psychobiological silly season-or-what happens when neurophysiological data become psychological theories. Journal of General Psychology, 1971, 84, 151-166.

Weisstein, N. Metacontrast. In D. Jameson \& L. M. Hurvich (Eds.), Handbook of sensory physiology (Vol. 7, part 4): Visual psychophysics. New York: Springer-Verlag, 1972.

\section{NOTE}

1. Because of the nature of the interface between the Optacon and the computer, and the mechanical characteristics of the tactile array, the mechanical vibration may last several milliseconds beyond the offset of the electrical signal.

(Manuscript received January 11, 1982; accepted for publication February 17, 1982.) 